\title{
Criminologie
}

\section{De la police au tribunal : formulation et cheminement des plaintes portées devant la Cour du Québec, Chambre criminelle et pénale}

\author{
Marie-Marthe Cousineau et Guy Cucumel
}

Volume 24, numéro 2, 1991

Regard sur les tribunaux

URI : https://id.erudit.org/iderudit/017309ar

DOI : https://doi.org/10.7202/017309ar

Aller au sommaire du numéro

Éditeur(s)

Les Presses de l'Université de Montréal

ISSN

0316-0041 (imprimé)

1492-1367 (numérique)

Découvrir la revue

Citer cet article

Cousineau, M.-M. \& Cucumel, G. (1991). De la police au tribunal : formulation et cheminement des plaintes portées devant la Cour du Québec, Chambre criminelle et pénale. Criminologie, 24(2), 57-79.

https://doi.org/10.7202/017309ar
Résumé de l'article

To date, no one really knows exactly how cases go through the maze of legal proceedings after being filed. Based on a sampling ofl 795 requests to undertake legal proceedings presented by the Montreal Urban Community Police Service to "la Cour du Québec, chambre criminelle et pénale" (Quebec Criminal Court) and dealt with by this tribunal in 1989, the authors take a close look at how the intervention of the police (usual access to the judiciary system) and the intervention of the tribunal (decision making element of prime importance) are linked. Greater importance is given to three particular aspects of this matter. First, the authors consider how the police and the tribunal formulate the accusation. This allows them to throw a new light on the problem of over charging. Secondly, the authors reflect on the equally questionable issue of temporary custody at both the police and the tribunal levels. Finally, the authors conclude by examining the processing of cases at the tribunal level. 
DE LA POLICE AU TRIBUNAL: FORMULATION ET CHEMINEMENT DES PLAINTES PORTÉES DEVANT LA COUR DU QUÉBEC, CHAMBRE CRIMINELLE ET PÉNALE ${ }^{1}$ Marie-Marthe Cousineau ${ }^{2}$, Guy Cucumel ${ }^{3}$

To date, no one really knows exactly how cases go through the maze of legal proceedings after being filed. Based on a sampling of 1795 requests to undertake legal proceedings presented by the Montreal Urban Community Police Service to "la Cour du Québec, chambre criminelle et pénale" (Quebec Criminal Court) and dealt with by this tribunal in 1989, the authors take a close look at how the intervention of the police (usual access to the judiciary system) and the intervention of the tribunal (decision making element of prime importance) are linked. Greater importance is given to three particular aspects of this matter. First, the authors consider how the police and the tribunal formulate the accusation. This allows them to throw a new light on the problem of over charging. Secondly, the authors reflect on the equally questionable issue of temporary custody at both the police and the tribunal levels. Finally, the authors conclude by examining the processing of cases at the tribunal level.

Le Canada et, par conséquent, le Québec sont régis par un nombre impressionnant de lois et de règlements que nul n'est censé ignorer ${ }^{4}($ !) et dont la transgression, pour une bonne part, est susceptible de se traduire par des sanctions de nature pénale. En 1976, la Commission de réforme du droit du Canada recensait quelque 700 articles au Code criminel, 20000 infractions aux lois fédérales, 20000 infractions aux lois provinciales, sans compter une

1. La réalisation de l'étude dont il est question dans le cadre de cet article est rendue possible grâce à une subvention décernée par le Fonds FCAR, volet Action spontanée. Le masculin est utilisé comme générique neutre afin d'alléger le texte. Les auteurs tiennent à remercier Danielle Laberge, professeure titulaire au département de sociologie de l'UQAM et co-directrice du Groupe de recherche et d'analyse sur les politiques et les pratiques penales, le GRAPPP, pour sa lecture attentive et ses commentaires judicieux.

2. Chercheure de niveau post-doctoral au département de sociologie de l'UQAM, membre du Groupe de recherche et d'analyse sur les politiques et les pratiques pénales, C.P., 8888, Succ. A, Montréal (Québec) H3C 3P8.

3. Chercheur de niveau post-doctoral au département de sociologie de l'UQAM, membre du Groupe de recherche et d'analyse sur les politiques et les pratiques pénales, C.P. 8888. Succ. A, Montréal (Québec) H3C 3P8.

4. "Nul n'est censé ignorer la loi»: cette maxime est officiellement sanctionnée, a tout le moins en ce qui concerne les lois publiques de la province. $\grave{A}$ ce sujet et au sujet des exceptions à cette règle, voir G. Létourneau et P. Robert (1990), Code de procédure pénale du Québec annoté, Montréal, Wilson et Lafleur Ltée. 
multitude d'infractions aux règlements municipaux ${ }^{5} »$. Dans les circonstances, il n'est pas surprenant de constater qu'au Québec, chaque année, des milliers de justiciables ${ }^{6}$ sont traduits devant les cours de justice pour avoir contrevenu à l'une de ces lois ou à l'un de ces règlements qui sanctionnent toute une gamme de comportements de nature aussi diverse que le vagabondage, les paris illicites, la prostitution, la fraude, le vol simple, l'introduction par effraction, le vol qualifié, l'homicide involontaire ou le meurtre avec préméditation... Certains sont libérés des accusations retenues contre eux; d'autres en sont acquittés; d'autres enfin plaident coupables (la majorité) ou sont reconnus coupables. Pour ces deux derniers groupes de contrevenants, toute une gamme de sanctions sont possibles: probation, travaux communautaires, ordonnance de dédommagement ou de restitution, amende, prison...

Concernant ces réalités, on a tendance à croire que tout est connu, fiché, mesuré. Il s'agit de vouloir s'intéresser d'un peu plus près à l'activité des tribunaux criminels pour se voir rapidement confronté à un manque de données empiriques désarmant pour qui cherche à comprendre les mécanismes à l'œuvre dans la construction des affaires de nature pénale. Outre les rapports annuels, qui rendent compte du nombre de plaintes portées à la police, du nombre de demandes d'intenter des procédures adressées à la cour, du nombre de causes entendues devant les tribunaux, du nombre de condamnations, de sentences rendues, d'admissions enregistrées dans les institutions carcérales, de dossiers gérés par la probation, etc., et, surtout, des budgets alloués et dépensés pour les fins de l'administration de la justice, aucune information plus structuree, plus parlante n'est, à l'heure actuelle, accessible au chercheur.

Partant de ces informations, on peut tenter d'imaginer la configuration du flux de même que les trajectoires des individus à travers l'ensemble du système pénal. Toutefois, une telle stratégie, si elle permet d'esquisser un portrait partiel et minimal de l'activité du système pénal, ne permet absolument aucune inférence sérieuse quant aux types de cheminements judiciaires que connaissent les causes. Seul le suivi d'une cohorte d'individus? , à travers l'en-

5. Commission de réforme du droit du Canada (1976), Notre droit pénal, Ottawa, Approvisionnements et services, rapport $n^{\circ} 3$. Une citation désormais célèbre. Il reste par ailleurs établi qu'en matière pénale il n'y a pas de crime sans texte d'incrimination: «Nullum crimen, nulla poena sine lege.» (Op. cit., note 4, p. 4.)

6. Au sens où nous l'employons ici, le terme «justiciable» désigne toute personne susceptible de faire l'objet d'une poursuite pour avoir contrevenu à l'une des lois ou à l'un des règlements en vigueur au Québec, commettant ainsi une infraction pénalisable. Nous entendons donc, dans les circonstances et à moins de cas exceptionnels (il arrive que des juvéniles soient traduits devant les tribunaux pour adultes), tout citoyen légalement majeur.

7. Le terme «cohorte» est prís ici dans son acception la plus large, qui désigne un groupe, un échantillon, une population caractérisés par un certain nombre d'attributs communs, en l'occurrence le fait d'avoir été l'objet d'une demande d'intenter des procédures 
semble des étapes judiciaires, nous paraît pouvoir remplir les conditions d'une telle étude qui permettrait de bien cerner les mécanismes de circulation à l'ceuvre dans la prise en charge pénale des justiciables mis en accusation.

C'est donc directement à la source, et non seulement dans les rapports officiels, qu'il faut aller puiser les données concernant le cheminement des causes, et partant des suspects, à l'intérieur du processus judiciaire. C'est à cette tâche que nous nous sommes consacrés. L'étude que nous avons entreprise consiste à suivre une cohorte de justiciables à partir du moment où une demande d'intenter des procédures est formulée par la police jusqu'à l'issue de la cause au tribunal. L'étude est actuellement en cours de réalisation. Les résultats, dont nous livrons ici un premier aperçu, sont issus d'une série d'analyses initiales, essentiellement des tris à plat et des tableaux de contingence effectuées sur les données en regard d'hypothèses bien précises.

S'ils n'ont rien de très complexe, ces traitements ont déjà le mérite de lever le voile sur toute une gamme de pratiques qui, de notre point de vue, sont un peu trop allègrement l'objet de «on dit...», «il paraît que...», «on estime à...», aussi bien dans les conversations de salon que dans les réunions scientifiques, mais sur lesquelles, concrètement, bien peu de choses sont connues. Trois questions sont abordées dans le cadre nécessairement restreint de cet article. Dans un premier temps, nous nous intéressons à la formulation des accusations tant au niveau de la police qu'au niveau du tribunal. Cette première exploration des données nous permet de jeter un regard nouveau sur la question controversée de l'over charging. Dans un deuxième temps, nous abordons la question tout aussi problématique de l'usage de la détention provisoire, encore une fois aux deux niveaux, police et tribunal. Enfin, nous traitons plus particulièrement du traitement que connaissent les causes au niveau du tribunal. En filigrane de toute notre démarche se profile l'étude de l'articulation entre l'intervention de la police (porte d'entrée privilégiée du système judiciaire) et du tribunal (nœud décisionnel déterminant).

\section{LE DÉFI DE FAIRE CORRESPONDRE PLUSIEURS SOURCES DE DONNÉES}

L'étude de cette question telle que nous la concevons nous oblige à mettre directement en relation les informations provenant de deux sources de données différentes et indépendantes: d'une part, les données consignées à la police se rapportant à l'événement et, d'autre part, les informations provenant du

adressée en 1989 par le SPCUM (Service de police de la Communauté urbaine de Montréal) à la Cour du Québec, chambre criminelle et pénale du district judiciaire de Montréal. 
tribunal concernant le cheminement de la cause, du moment de la comparution jusqu'à l'issue des procédures. La complémentarité de ces deux sources de données s'avère essentielle à la réalisation des analyses que nous menons.

À la police, les demandes d'intenter des procédures nous renseignent sur: 1) le contrevenant (ses caractéristiques socio-démographiques: âge, sexe, lieu de résidence, statut civil, occupation, situation et casier judiciaire;2) le délit et les circonstances entourant sa commission: nature du délit, heure, jour, lieu de l'infraction, nombre, âge, sexe des victimes, lien victimeagresseur, usage de violence, de menaces, consommation d'alcool, de drogue, etc.; 3) les circonstances entourant l'arrestation.

Au tribunal, le plumitif informatisé nous informe quant au cheminement de l'individu, à partir du moment de sa première comparution devant le tribunal jusqu'à l'issue des procédures. Ces données concement essentiellement les caractéristiques légales ou judiciaires de l'affaire telles qu'elles se dessinent à chacune des étapes de la procédure. Parmi celles-ci, pour des raisons techniques liées aux traitements informatique et statistique, nous retenons strictement trois moments privilégiés de la procédure: la comparution, la décision sur la culpabilité de l'inculpé et la sentence. À chacune de ces étapes, nous nous intéressons aux accusations retenues (nombre, nature et modification des chefs d'accusation). Sur l'ensemble des étapes, nous traitons l'information concernant la décision déterminante dans le dossier ${ }^{8}$ (plaidoyer de culpabilité, inculpation, acquittement, libération) et, lorsqu'il y a lieu, la sentence sur chacun des chefs. Enfin, à chacune des étapes, nous portons une attention toute particulière au statut de l'inculpé (liberté inconditionnelle, liberté sous caution, détention préventive), de même qu'à l'évolution de ce statut.

L'échantillon traité dans cette étude est constitué de 1795 dossiers de cour initiés à la suite d'une demande d'intenter des procédures déposée par le SPCUM devant la Cour du Québec, chambre criminelle et pénale du district judiciaire de Montréal, traités par ce tribunal au cours de l'année 1989 et dont l'issue finale est connue. Cet échantillon, constitué suivant une procédure stratifiée non proportionnelle dans le but d'assurer une représentation suffisante des différents types d'événements portés par les policiers à l'attention du tribunal, a été retracé à la police ${ }^{9}$.

8. La décision déterminante est celle qui est considérée comme étant la plus significative en regard de l'issue de la cause. Cette donnée est construite à partir de choix conceptuels qui considèrent que la décision la plus marquante est celle de l'enregistrement d'un plaidoyer de culpabilité ou encore la reconnaissance judiciaire de la culpabilité de l'inculpé, suivie de l'acquittement et finalement de la libération du suspect sur l'ensemble des chefs d'accusation enregistrés à son dossier.

9. Les distributions de fréquences échantillonnales sont conformes aux distributions de fréquences du plumitif, sauf en ce qui a trait aux crimes causant la mort et aux agressions 
À notre connaissance, aucune étude de cette nature n'a encore été réalisée à ce jour. Le problème ne tient certainement au manque de bonne volonté, mais réside essentiellement, comme le note Robert ${ }^{10}(1977$, p. 16), dans le fait qu'il est fréquent que «les diverses statistiques d'un même système pénal adoptent des unités de compte différentes, qu'elles ne rapportent pas les mêmes renseignements sur les populations concernées» et surtout, ajoutonsnous, que jusqu'à tout récemment les données recueillies indépendamment pour le compte d'une agence ou d'une autre n'utilisaient pas les mêmes identificateurs, les mêmes référents pour désigner les individus pris en charge, de sorte qu'il se révélait à toutes fins utiles impensable de faire correspondre systématiquement une partie de l'information consignée à un endroit (par exemple à la police) avec une autre partie de l'information conservée ailleurs (à la cour, par exemple). L'informatisation grandissante des données (inégalement répartie toutefois à travers les différentes agences) semble susciter une conscience nouvelle du besoin d'uniformiser, sinon les modes de saisie, du moins les référents. Des efforts sérieux sont actuellement faits en ce sens ${ }^{11}$. La disparition définitive des dossiers physiques au profit des dossiers informatisés (appelée inévitablement à se concrétiser à plus ou moins long terme) devrait, croyons-nous, inciter définitivement les responsables des agences à s'entendre sur l'utilisation d'un référent commun permettant la communication inter-agences de l'information.

Il est par ailleurs une autre difficulté sur laquelle nous voulons insister ici. Les données compilées pour le compte des différentes agences le sont le plus souvent dans un but de gestion administrative et non de recherche sociologique, criminologique ou autre. En conséquence, une partie des données essentielles aux yeux des chercheurs (les données socio-démographiques, entre autres: scolarité, situation familiale, revenu, conditions d'emploi...) sont inva-

sexuelles graves, catégories pour lesquelles la totalité des événements a été retenue. La taille de l'échantillon permet d'assurer une marge d'erreur maximale de 2,8 pour cent avec un risque d'erreur de 1 pour cent. La surreprésentation des délits mentionnés plus haut, justifiée compte tenu de la problématique de l'étude, ne devrait poser aucun problème tant et aussi longtemps que l'on prend la précaution de ne faire aucune inférence sur la distribution des délits elle-même.

10. Ph. Robert (1977), «Les statistiques criminelles et la recherche : réflexions conceptuelles», Déviance et société, vol. 1, n 1, p. 3-27.

11. Soulignons à cet égard, entre autres, les efforts fournis par le Centre canadien de la statistique juridique qui, dans son plan de restructuration du programme DUC (programme de déclaration uniforme de la criminalité), vise désormais à assurer la continuité historique des statistiques de la criminalité et, encore plus particulièrement, les efforts conjoints du ministère de la Sécurité publique et des services correctionnels du Québec qui, dans la mise sur pied du nouveau système DACOR (système informatisé de gestion des dossiers administratifs correctionnels), prévoit l'utilisation d'un numéro de dossier unique permettant de reconstituer l'historique judiciaire et correctionnel d'un contrevenant et de connaître l'évolution de son dossier. 
riablement négligées au profit d'autres informations apparemment plus utiles pour le gestionnaire (taille, teint, couleur des yeux, marques distinctives, alias, non de jeune fille de la mère...). Cette situation, incontournable, gêne considérablement le chercheur qui se voit contraint de travailler avec des données beaucoup moins complètes qu'il ne l'avait imaginé. L'informatisation des données laisse un peu trop facilement présager de l'exhaustivité de celles-ci: on perd souvent de vue que les renseignements détenus par la machine, et consequemment les traitements qu'on peut en faire, sont strictement fonction de l'information qui a été saisie au départ, ni plus ni moins. L'ordinateur, faut-il le rappeler, n'invente pas l'information, il ne fait que la mémoriser et, suivant les directives du chercheur, la traiter.

En allant cueillir nous-mêmes nos données à la source, à même la demande d'intenter des procédures, nous avons réussi à pallier cette difficulté, du moins en partie, puisqu'il devenait possible de choisir, à notre convenance, les informations qui nous paraissaient les plus pertinentes en regard de notre problématique. Reste le problème des données non consignées sur le formulaire de demande d'intenter des procédures qui, lui, ne peut être contourné.

\section{QUELQUES REMARQUES PRÉLIMINAIRES BIEN CONNUES}

Les quelques remarques glissées ici n'ont rien de très original; elles ont déjà été formulées, documentées, publiées à de nombreuses reprises. Toutefois, on a encore, trop souvent pensons-nous, tendance à les perdre de vue. C'est pourquoi nous tenions à les reprendre ici, au moins sommairement, ceci afin de bien situer le cadre des analyses que nous effectuons.

La première concerne la portée des statistiques dites «de la criminalité» ou «criminelles». L'importance donnée à cette question se justifie par l'ampleur considérable du chiffre noir, «symbole irritant de cet hiatus existant entre l'infraction et sa prise en charge», selon l'expression de Zauberman ${ }^{12}$ (1982, p. 23). Landreville ${ }^{13}$ (1983, p. 24) remarque: «compte tenu de l'importance du contrôle social informel non-étatique et des autres modalités de contrôles sociaux étatiques, il n'est certes pas exagéré de dire que le système pénal ne gère qu'une infime partie des cas qui pourraient être de son ressort». Citant Denzin (1970), Landreville rapporte: «Few persons ever come in contact with the courts and legal institutions, but many persons are defined by

12. R. Zauberman (1982), «Renvoyants et renvoyés», Déviance et société, vol. 6, $n^{\circ} 1$, p. $23-52$.

13. P. Landreville (1983), Normes sociales et normes pénales; notes pour une analyse socio-politique des normes, Montréal, Université de Montréal, Les Cahiers de l'École de criminologie, cahier $n^{\circ} 12$. 
some set of others as deviant $[\ldots]$ Publicly to acclaim a person a deviant and move him into a formal treatment agency is an extreme act, indeed ${ }^{14}[\ldots]$ » À l'instar de Dezin, plusieurs auteurs ont noté et commenté le fait qu'il ne suffit pas qu'un acte soit commis, qu'une action répréhensible - au sens de la loi - soit posée d'abord pour que le système pénal en soit informé et, ensuite, pour qu'il accepte de s'en saisir ${ }^{15}$. Les travaux de Robert et Faugeron ${ }^{16}$, entre autres, ont permis de montrer qu'entre la commission d'un acte criminalisable et sa reconnaissance par le système pénal joue un mécanisme intermédiaire complexe, la reportabilité, fondée par la combinaison de la visibilité de l'acte (variant selon la sorte et le contexte de l'événement, compte tenu de la présence de témoins, et selon la position sociale de l'actant ${ }^{17}$ ) et du renvoi (la décision de faire appel à une agence de contrôle social dans le but de régler une situation jugée problématique).

Par ailleurs, notent encore Faugeron et al. ${ }^{18}$ (1977, p. 19). «Parmi les personnes et les affaires qui lui parviennent, constituant sa matière première potentielle, chaque appareil de contrôle va opérer un tri. Il en gardera certaines et en rejettera, plus ou moins précocement, d'autres. Il agencera progressivement ce qu'il aura conservé, mettant en relief certains éléments, en ignorant d'autres, pour parvenir à un produit fini conforme à sa logique de fonctionnement, donc, finalement, à sa fonction sociale ${ }^{19}$.» En définitive, constatent

14. N.K. Denzin (1970), «Rules of Conduct and the Study of Deviant Behavior: Some Notes on the Social Relationship"; chap. V in J.D. Douglas (ed), Deviance and Respectability. The Social Construction of Moral Meanings, New York, Basic Books Inc.

15. Entre autres A.K. Bottomley (1973), Decisions in the Penal Process, South Hackensack, Fred B. Rothman et Co.; Ph. Robert (1977). loc cit., note 10; A.K. Bottomley et K. Pease (1986), Crime and Punishment: Interpreting the Data, Philadelphie, Open University Press; M.R. Gottfredson et D.M. Gottfredson (1988), Decision Making in Criminal Justice: Toward the Rational Exercise of Discretion, New York, Plenum Press.

16. Parmi les travaux réalisés par le groupe de Robert et Faugeron, voir entre autres: Ph. Robert, Th. Lambert et C. Faugeron (1976), Image du viol collectif et reconstruction d'objet, Paris, Médecine et Hygiène; C. Faugeron, M. Fichelet et Ph. Robert (1977), Le Renvoi du déviant: des modes informels aux systèmes institutionnels de contrôle de la déviance, Paris, AERDU; Ph. Robert, (1977), loc. cit., note 10.

17. À ce sujet, voir, entre autres, D. Chapman (1968), Sociology and the Stereotype of the Criminal, Londres, Tavistock Publications. La discussion de Chapman autour de la notion de privacy, entre autres, mérite attention.

18. Op. cit., note 16.

19. Ce qui, d'ailleurs, fait dire à Hulsman (1982, p. 3): «Le processus de bureaucratisation et de professionnalisation qui se đéveloppe dans nos pays opère un phénomène de réduction des événements, de sorte que ceux dont traitent les administrations n'existent pas dans la réalité; et lorsqu'un problème entre dans le système pénal, il n'est pas traité tel qu'il est vécu, mais tel qu'il est artificiellement enregistré et transmis par le dit système. Ainsi se trouvent qualifiés crimes ou délits des situations-problèmes que les seuls intéressés, spontanément, n' auraient généralement pas envisagées ainsi. » In J. Bernat de Celis (1982), «Les grandes options de la politique criminelle: la perspective de Louk Hulsman», Les Archives de politique criminelle, $\mathrm{n}^{\circ}$ 5, p. 13-60. 
Bottomley et Pease (1986, p. 3): «The crime rate is the product of a series of decisions taken by victims or witnesses of crime, by police officers, or by offenders who may make the police aware of earlier crimes by admitting them together with the offence for which they are apprehended ${ }^{20}$."

Il ne saurait donc être question de présenter nos résultats comme reflétant l'état de la criminalité et de son traitement au Québec. Même si on parvenait avec suffisamment de précision à corriger l'échantillon pour le faire correspondre proportionnellement à ce que l'on sait (ou à tout le moins à ce que l'on estime savoir, que ce soit à partir des sondages de victimisation ou encore des enquêtes de délinquance auto-révélée) de l'ensemble de la criminalité, rapportée ou non, il reste des limites à nos données qui ne doivent pas être perdues de vue.

Une première limite de l'étude provient du fait qu'on s'intéresse aux plaintes inscrites par la police au tribunal. La police, bien qu'elle en constitue la porte d'entrée par excellence, ne représente pas la seule source d'alimentation du système pénal. Il est en effet possible, soit pour un particulier, ce qui est rarement le cas, soit pour une autre instance administrative, de porter directement plainte au tribunal. Zauberman ${ }^{21}$ (1982) constate qu'en effet le renvoi (vers le pénal et, en certaines occasions, directement au tribunal) ne provient pas toujours du groupe primaire (les citoyens), mais qu'il existe aussi des renvoyants spécialisés (d'autres réseaux de contrôle social allant des services sociaux aux polices privées) qui contribuent, dans des proportions variables, à l'alimentation du système pénal. De fait, poursuit l'auteur, il s'agit même parfois d'un processus en cascade où le groupe primaire renvoie à un acteur spécialisé, au niveau du contrôle social étatique ou non étatique, qui lui-même renverra à un autre réseau de contrôle social... en l'occurrence le pénal. Il n'est pas exclu que la prise en compte de ces autres sources d'alimentation des tribunaux pénaux, et plus particulièrement des cours criminelles, contribue à dessiner une distribution significativement différente des «affaires» traitées par cette instance. On peut assez facilement entrevoir que la représentation du nombre des fraudes, par exemple, augmenterait de manière importante si on tenait compte des plaintes portées directement à l'attention de la cour par des instances administratives étatiques ou privées. De même la représentation du nombre des vols à l'étalage serait-elle reprécisée par les incidents de cette nature rapportés sans intermédiaire au tribunal par les services de sécurité privés.

20. Op. cit., note 15 .

21. Loc, cit., note 12 . 
Par ailleurs, la police jouit du pouvoir discrétionnaire de déjudiciariser les situations pour lesquelles elle juge que le recours au pénal n'est pas essentiel ou justifié dans les circonstances. D'autre part, elle peut, compte tenu encore une fois des pouvoirs qui lui sont conférés, juger une plainte non fondée et décider de clore le dossier sans aller plus avant ${ }^{22}$. Il s'opère donc, dès l'étape de l'intervention policière, un tri des affaires qui seront portées à l'attention du tribunal.

Soulignons enfin qu'il existe, au Québec, plusieurs niveaux de tribunaux susceptibles d'entendre les causes criminelles ${ }^{23}$ (cours municipales, Cour du Québec, Cour supérieure de juridiction criminelle, pour ne nommer que les plus importantes en termes de nombre de cas traités). Lorsqu'il s'agit d'infractions sommaires ou mixtes, les policiers peuvent décider de faire appel soit aux tribunaux municipaux, soit à la Cour du Québec, chambre criminelle et pénale. La décision quant à l'instance est souvent tributaire des ressources disponibles ${ }^{24}$. Dans le district judiciaire de Montréal, la pratique veut que certains délits soient quasi systématiquement référés à la cour municipale. Ainsi en est-il, entre autres, des infractions de mœurs (prostitution, proxénétisme), des paris illicites, des délits que nous qualifierons de «délits de comportement» (ivresse sur la voie publique, tapage, vagabondage...) et de la conduite avec facultés affaiblies, ce qui explique qu'on trouve ces infractions si peu représentées dans notre échantillon. Les cas d'inceste, pour leur part, sauf exception, sont traités au niveau du tribunal de la jeunesse.

En définitive, il faut prendre les données que nous traitons pour ce qu'elles sont, c'est-à-dire un échantillon représentatif des causes issues des demandes d'intenter des procédures formulées par le SPCUM à l'endroit de la Cour du Québec, chambre criminelle et pénale du district judiciaire de Montréal, et ne pas chercher à leur faire dire - comme il ne faudrait d'ailleurs jamais chercher à le faire - ce qu'elles ne peuvent pas dire. Il ne faut par ailleurs pas limiter injustement la portée des conclusions que nous en tirons. Ces données, compte tenu de l'importance de la police comme source d'alimentation de la Cour du Québec, permettent de donner un bon aperçu du fonctionnement de cette Cour et des affaires traitées par elle.

22. Concernant les pouvoirs des policiers en matière de déjudiciarisation voir, entre autres, M. Gabias, G. Lambert et J. Trudel (1987), Droit pénal général et pouvoirs policiers, Montréal, Modulo Éditeur.

23. À ce sujet, voir D. Saint-Laurent (1986), Principes de droit constitutionnel et pénal, Montréal, Modulo Éditeur.

24. Op. cit., note 22. 


\section{DE LA POLICE AU TRIBUNAL: FORMULATION D'UNE ACCU- SATION}

Nos données permettent d'examiner la mise en forme des accusations premièrement par les policiers puis par les procureurs de la Couronne et des modifications survenant dans le passage d'une étape à l'autre. Ce faisant, la question de l'over charging, cette pratique qui consisterait, d'abord pour les policiers puis pour les procureurs de la Couronne, à gonfler indûment soit le nombre, soit la gravité des accusations portées contre l'inculpé se trouve nécessairement posée. Nombreux sont les auteurs qui dénoncent cette situation jugée inacceptable, arguant qu'il s'agit d'une façon malhonnête de se procurer une marge de négociation suffisante et d'amener l'accusé à plaider coupable en lui faisant miroiter des avantages qui en réalité n'en sont pas (Ericson et Baranek, $1982^{25}$ ). Différents indices nous portent à croire que l'importance de cette pratique serait, en de nombreuses circonstances, largement exagérée.

En effet, l'examen statistique des dossiers de notre échantillon montre que le nombre d'accusations par dossier s'élève à 2,3 chefs en moyenne, ce qui, de notre point de vue, ne représente pas une surcharge aussi dramatique qu'on a pu le laisser entendre. Cette donnée doit toutefois être examinée suivant le type d'accusation principale définissant la nature de chaque dossier. Ce faisant, on s'aperçoit que certains événements résultent dans l'enregistrement d'un nombre d'accusations supérieur à la moyenne, entre autres les dossiers d'agressions sexuelles graves (moyenne: 4,1 ) de même que ceux de vols qualifiés (moyenne: 3,3 ), alors qu'au contraire il est certains délits pour lesquels le nombre de chefs d'accusation inscrits au dossier se révèle en deçà de la moyenne (voir tableau 1). En examinant de plus près la configuration des accusations multiples, on constate qu'il s'agit beaucoup plus souvent d'une série d'accusations se rapportant au même événement (par exemple: complot, possession d'outils de cambriolage, introduction par effraction, recel) que đ'une accumulation de délits de même nature que l'on choisit de regrouper en un seul dossier de cour pour les traiter simultanément (une suite d'agressions sexuelles, par exemple). Ces deux types de situations donneront potentiellement lieu à des pratiques d'over charging différentes.

Par ailleurs, on constate que, dans une grande majorité de cas $(89,5 \%)$, l'accusé plaide coupable à l'accusation principale portée dans le dossier. Les réductions d'accusation, du moins la réduction des accusations portées dès le départ par le procureur de la Couronne dans le dossier de cour, sont

25. R. Ericson et P. Baranek (1982), The Ordering of Justice: A Study of Accused Persons as Dependents in the Criminal Process, Toronto, University of Toronto Press. 
Tableau 1

Distribution du nombre de chefs d'accusation par dossier selon l'accusation principale définissant la nature du dossier

$\begin{array}{lr}\text { Crimes contre la personne causant la mort } & 1,2 \\ \text { Agressions sexuelles graves } & 4,1 \\ \text { Autres crimes graves contre la personne } & 2,2 \\ \text { Vols qualifiés } & 3,3 \\ \text { Voies de fait sur un policier } & 2,4 \\ \text { Autres crimes contre la personne } & 1,7 \\ \text { Vol et recel } & 2,7 \\ \text { Introduction par effraction } & 2,5 \\ \text { Fraudes, fausses représentations } & 2,9 \\ \text { Vandalisme et autres crimes contre les biens } & 1,5 \\ \text { Désobéissance à la cour } & 2,2 \\ \text { Drogue } & 1,9 \\ \text { Complots et tentatives } & 1,8 \\ \text { Possession d'arne } & 1,2 \\ \text { Autres } & 1,3\end{array}$

relativement rares, comme en témoigne le tableau 2 qui met en présence accusation principale et accusation déterminante ${ }^{26}$ dans le dossier ${ }^{27}$. On pourrait arguer ici que la réduction des accusations se fait ailleurs, avant que l'acte d'accusation ne soit définitivement arrêté, soit entre le moment où le policier formule la demande d'intenter des procédures et le moment où le procureur de la Couronne s'en saisit. On pourrait alors parler d'over charging policier.

Il est possible, à partir de nos données, d'examiner l'adéquation entre les accusations enregistrées par la police au niveau de la demande d'intenter des procédures, c'est-à-dire celles qui sont suggérées par le policier au procureur de la Couronne, et la formulation que ce dernier choisit de retenir pour la poursuite des procédures à la cour. L'écart est ici plus grand, sans toutefois être dramatique. De façon plus précise, notons que la distribution de nos données montre que, dans 84,4 pour cent des cas, le libellé des accusations enregistrées par les policiers au niveau de la demande d'intenter des procédures correspond exactement à celui retenu par les procureurs de la Couronne au

26. L'accusation principale dans le dossier est celle qui correspond à l'événement le plus prégnant en termes de gravité. L'ordre de gravité que nous privilégions est défini conceptuellement dans le cadre de l'étude. L'accusation déterminante est celle sur laquelle la décision déterminante, telle que nous la définissons plus haut (note 8), est rendue.

27. Mentionnons qu'il est plausible ici de considérer la distribution des délits comme de niveau ordinal, ceux-ci ayant été classés suivant un ordre de gravité que nous avons conceptuellement choisi de prioriser. À ce titre, il nous est possible, au moins à titre indicatif, d'estimer la force de la relation existant entre les deux variables (accusation principale et accusation déterminante dans le dossier) à partir du Tau B de Kendall qui est ici de .94 $(\mathrm{p}>.0000)$, donc assurément significatif. 
Tableau 2

Rapport entre l'accusation principale et l'accusation déterminante dans le dossier de cours

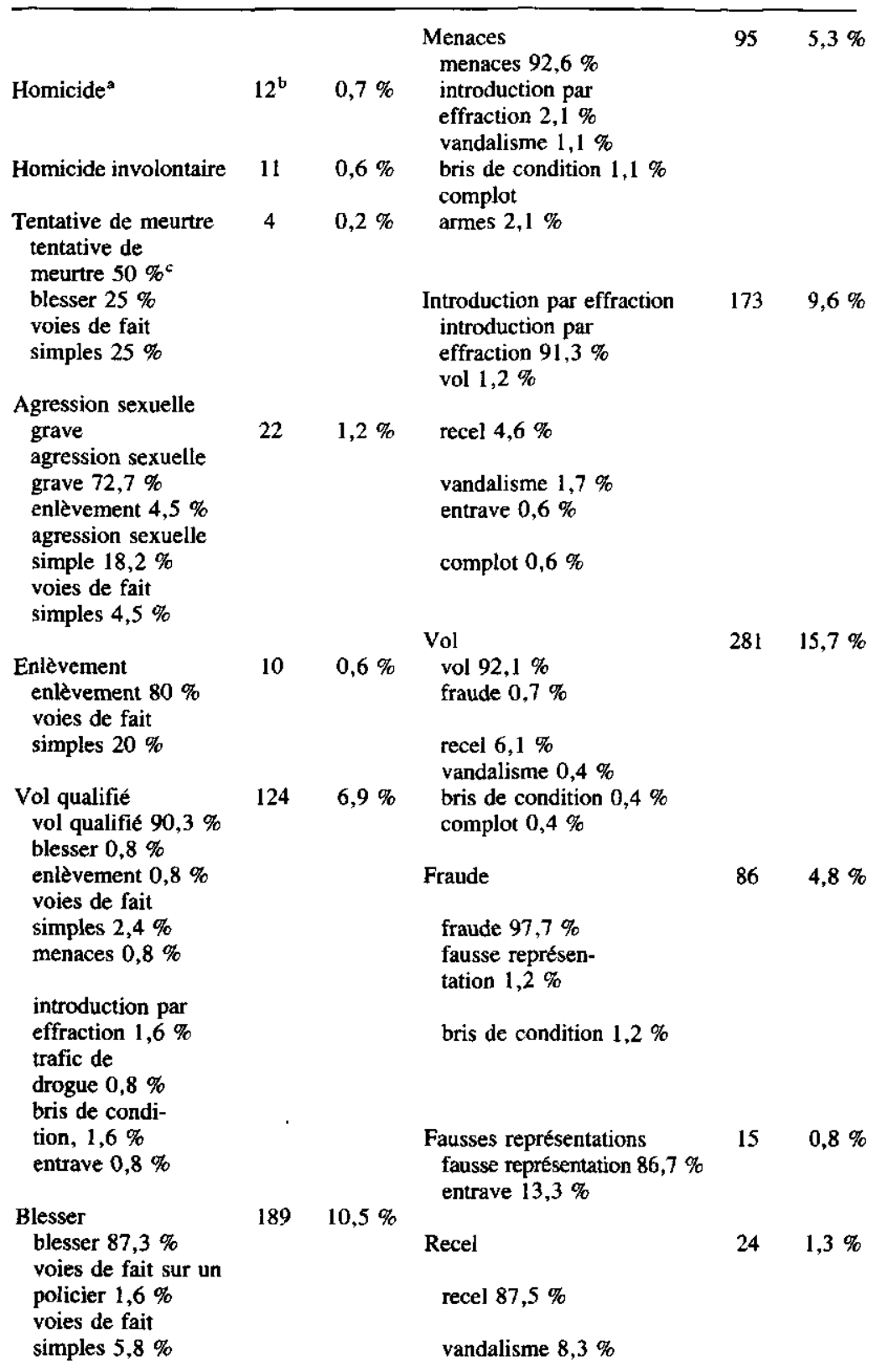


menaces $1,1 \%$

introduction par

effraction $1,6 \%$

vol $0,5 \%$

possession de

drogue $1,1 \%$

armes $1,1 \%$

\section{Agression sexuelle simple \\ Voies de fait sur un policier voies de fait sur un policier $89,5 \%$ voies de fait simples $5,3 \%$ menaces $2,6 \%$ fraude $2,6 \%$ \\ Voies de fait simples voies de fait simples $95,2 \%$ menaces $2,7 \%$ introduction par effraction $0,3 \%$ vandalisme $1,4 \%$ bris de condi- tion $0,3 \%$}

\section{Armes} \\ bris de condition $4,2 \%$ \\ complot $4,2 \%$ \\ Vandalisme \\ vandalisme $97,6 \%$ \\ nuisance publique $1,2 \%$ \\ $83 \quad 4,6 \%$ \\ complot $1,2 \%$ \\ $38 \quad 2,1 \%$ Désobéissance à la cour \\ $97 \quad 5,4 \%$ \\ 291 \\ $16,2 \%$ \\ Trafic de drogue \\ $84 \quad 4,7 \%$ \\ Possession de drogue \\ $52 \quad 2,9 \%$ \\ Entrave à un agent de la paix \\ $23 \quad 1,3 \%$ \\ entrave $91,3 \%$ \\ complot $8,7 \%$ \\ Complot et tentative \\ $28 \quad 1,6 \%$ \\ complot $96,4 \%$ \\ armes $3,6 \%$ \\ Autres \\ $11 \quad 0,6 \%$
}

accusation principale dans le dossier de cour.

b Nombre et pourcentage de dossiers dans l'échantillon par catégorie d'accusation principale dans le dossier de cour.

c Accusation déterminante en rapport avec l'accusation principale dans le dossier de cour.

moment de formuler définitivement l'acte d'accusation. Cette proportion nous apparaît tout de même relativement importante ${ }^{28}$. Elle témoigne du fait qu'il existe, somme toute, peu de distance entre les vues du policier et celles du procureur de la Couronne concernant un même événement. Reste 15,6 pour cent des cas pour lesquels les accusations diffèrent. Cette variation s'observe dans un sens comme dans l'autre: soit que l'accusation formulée par le procureur de la Couronne paraît plus importante en termes de gravité (voies de fait simples passées à voies de fait graves, introductions par effraction ou vandalisme devenus vol qualifié, fausses représentations changées pour des introductions par effraction, par exemple) ou, au contraire, que ce dernier paraît

28. De la même façon et pour les mêmes raisons qu'énoncées plus haut (note 27), le calcul du Tau B de Kendall peut être appliqué ici. Ce dernier se chiffre à .84 (p. $>.0000$ ), témoignant encore une fois d'une liaison fortement significative entre les deux variables étudiées. 
diminuer la portée de l'accusation formulée au niveau policier (homicides modifiés pour devenir des homicides involontaires, tentatives de meurtre ou agressions sexuelles simples enregistrées comme des voies de fait simple...).

Au terme des analyses mettant en rapport ces deux distributions (accusations formulées par la police et accusations portées au niveau de la poursuite), on constate que, dans les faits, il arrive quasi aussi souvent que les accusations de la police soient réduites par la Couronne $(6,8 \%$ des cas) ou, au contraire, qu'elles soient amplifiées par elle ( $8,8 \%$ des cas).

Différentes explications peuvent être apportées pour tenter de justifier ces écarts. D'une part, il arrive qu'entre le moment où la demande d'intenter des procédures est adressée à la Cour et le moment où elle est effectivement traitée, l'enquête policière a permis de mettre au jour de nouveaux éléments qui motivent l'enregistrement d'autres accusations que celles initialement prévues. Par ailleurs, le procureur de la Couronne peut, à l'étude de la preuve, juger qu'il y a matière à engager les procédures sur des accusations plus ou moins en rapport avec celles rapportées par les policiers. Enfin, dans la construction de sa preuve, la poursuite peut ne pas être à même de réunir tous les éléments nécessaires à l'établissement de la culpabilité de l'inculpé aux accusations telles que définies par les policiers. Par exemple, le procureur peut se voir confronté au refus de certains témoins essentiels à l'établissement de sa preuve de se présenter devant la cour, ce qui est encore courant dans les cas de violence conjugale, ou il peut arriver qu'une preuve d'identification se révèle non concluante...

La question reste entière de savoir si cette assez forte concordance des accusations définies dans un premier temps par la police puis enregistrées plus tard sur l'acte d'accusation formulé par le procureur de la Couronne dénote une véritable convergence de vues des policiers et des procureurs. Cette convergence d'idées témoignerait alors soit du fait qu'il existe une façon somme toute évidente de définir un événement criminalisable suivant la catégorie légale qui le recouvre, soit encore du fait que policiers et procureurs partagent une même conception de l'«événement type», le normal crime tel que défini par Sudnow ${ }^{29}$ (1964). Ou peut-être cette apparence d'unité dans les décisions découlerait-elle plutôt de la prédétermination de l'aval par l'amont et, inversement, de l'amont vers l'aval de l'action des différents intervenants impliqués dans le traitement des événements judiciarisés en fonction de leurs attentes vis-à-vis l'action des autres intervenants. Ce phénomène a été largement décrit et commenté, principalement dans le cadre des travaux

29. D. Sudnow (1964), «Normal Crimes: Sociological Features of the Penal code in a Public Defender Office», Social Problems, p. 255-276.. 
de Robert et Faugeron ${ }^{30}$. Ainsi, précise Robert ${ }^{31}$ (1977, p. 13), quand une agence prend une décision, elle anticiperait ce qu'elle pense être la réaction probable des étages ultérieurs. De fait, la police tiendrait compte de ce qu'elle pense être la politique criminelle du ministère public : systématiquement elle renverrait certaines affaires, mais elle en classerait définitivement d'autres ou les conserverait pour en traiter officieusement. D'un autre côté, le procureur de la poursuite, souvent dépassé par le nombre important de dossiers qu'il doit traiter au cours d'une même journée, aura tendance à s'en remettre à l'évaluation đu policier. Le débat est loin d'être tranché.

\section{DE LA POLICE AU TRIBUNAL: L'USAGE DE LA DÉTENTION}

L'usage de la détention, faut-il le rappeler, n'est pas exclusivement limité aux situations où un justiciable a été condamné: des personnes peuvent être incarcérées avant même que leur culpabilité n'ait été légalement reconnue.

La police, dans un premier temps, possède le pouvoir de détenir un individu suspect dans le but de le contraindre à comparaître devant un tribunal afin d'y être jugé. La décision de mettre sous garde peut se poursuivre jusqu'au moment de la comparution de l'inculpé devant le tribunal. Toutefois, suivant les circonstances et suivant qu'il ait été arrêté avec ou sans mandat, le suspect peut être remis en liberté soit par le policier, soit par un fonctionnaire responsable ${ }^{32}$. Au moment de sa comparution devant le tribunal, le juge peut 1) exiger que la mise sous garde de l'individu imposée par la police soit maintenue pour la poursuite des procédures; 2) décider de remettre en liberté le suspect avec ou sans conditions ou en exigeant ou non le versement d'une caution; ou encore 3) ordonner la mise sous garde d'un inculpé qui s'était présenté libre à sa comparution. L'ensemble de ces situations se traduit par autant de parcours potentiels différents pour les individus traduits devant le tribunal. Certains de ces parcours risquent de se révéler pénalisants pour les inculpés. Ainsi, remarquait déjà Friedland (1965), parlant de l'inculpé détenu provisoirement: "He is made to look like a convict and is treated like one. Further, the magistrate may not only be less inclined to find a "reasonable doubt" in the case of a person already in custody, but he may also be less hesitant to send such a person back to jail than to send him there initially ${ }^{33}$." Friedland insiste sur le fait que la détention provisoire «directly or indirectly,

30. Op. cit., note 16 .

31. Loc. cit., note 10.

32. Voir à ce sujet C. Cardinal (1989), Police et comparution devant le tribunal : différenciation sociale, légale et sexuelle, Montreal, Les Cahiers du GRAPPP, cahier $\mathrm{n}^{\circ} 8$.

33. Friedland (1965), Detention Before Trial: A Study of Criminal Cases Tried in the Toronto Magistrates's Courts, Toronto, University of Toronto Press. 
affects the accused's ability to engage counsel, hinders his attempt to present a proper defence, and increases the likelihood that he will be sentenced to imprisonment if convicted». Bref, Friedland, tout comme bon nombre d'auteurs ${ }^{34}$, dénonce l'influence négative de la détention provisoire sur l'issue des procédures et, le cas échéant, sur la sentence ${ }^{35}$.

Or, malgré ces constats et malgré les recommandations du Comité canadien de la réforme pénale et correctionnelle, lesquelles datent déjà de 1969 et ont été confirmées ultérieurement à diverses reprises par la Commission de réforme du droit du Canada ${ }^{36}$, recommandations voulant que «l'on s'efforce de réduire, dans toute la mesure du possible, l'arrestation et la détention sans nécessité des personnes suspectes» et que l'on maintienne en détention uniquement «lorsqu'une telle mesure est absolument indispensable pour la protection de la société ${ }^{37}{ }^{\prime}$, on constate qu'encore aujourd'hui on utilise massivement, il faut bien le dire, les mesures de détention provisoire.

Une étude préalable nous faisait conclure qu'en ce qui a trait aux admissions dans les établissements de détention provinciaux, les admissions de prévenus représentaient 48,6 pour cent, soit près de la moitié du total des admissions $^{38}$.

Nos données permettent de mieux cerner, d'une part, les pratiques policières $d$ 'incarcération avant comparution et, $d$ 'autre part, les décisions prises par les juges en regard de l'incarcération avant jugement. Le tableau 3 montre que seule une petite minorité $(5,7 \%)$ des suspects appréhendés par les policiers ne seront jamais arrêtés et comparaitront en liberté devant le tribunal;

34. Goldkamp (1979) cite à cet effet les études de Foote (1954); Alexander et al. (1958); Ares et al . (1963); Attorney General's Committee on Poverty and Federal Criminal Justice (1963); Rankin (1964); Single (1972); Brockett (1973); Landes (1974). (Voir J.S. Goldkamp, (1979), Two Classes of Accused: A Study of Bail and Detention in American Justice, Cambridge, Ballinger Publishing Co.)

35. Goldkamp $(1979$, p. 33$)$ conclut pour sa part: «the relationship between pre-trial custody and later judicial outcomes [... ] may or may not be shown to be spurious - depending on the kind of decision and the place of that decision in the criminal process. The "verdict", then, must be "it depends" ". Toutefois, Goldkamp reconnait qu' indépendamment de la nature du lien qui s'établit entre le statut de l'inculpé et l'issue des procédures, que celui-ci soit direct (causal model) ou indirect (spurious model), il reste que les contrevenants détenus provisoirement sont plus lourdement sanctionnés que ceux qui se présentent libres devant le tribunal. Op. cit., note 32 .

36. Voir sur cette question, entre autres, LArrestation, document de travail 41 , et Les Mesures assurant la comparution, la mise en liberté provisoire et la détention avant le procès, document de travail 57, Ottawa, Commission de réforme du droit du Canada.

37. Rapport du Comité canadien de la réforme pénale et correctionnelle (Rapport Ouimet) (1969), Justice pénale et correction : un lien à forger, Ottawa, Imprimeur de la Reine.

38. Cousineau et al. (1988), Mémoire présenté au Comité permanent de la justice et du Solliciteur général de la Chambre des communes du Canada, Montréal, Les Cahiers du GRAPPP, cahier $\mathrm{n}^{\circ} 4$. 
près du quart $(24,0 \%)$ seront d'abord arrêtés, amenés au poste puis relâchês par un fonctionnaire responsable; 4,2 pour cent des suspects n'auront pas été retracés au moment où la demande d'intenter des procédures est déposée au tribunal, un mandat d'arrestation étant émis à leur encontre. Les données de la cour montrent que la très grande majorité d'entre eux $(94,7 \%)$ auront néanmoins été écroués au moment de comparaître devant le tribunal. Enfin, près des deux tiers des contrevenants interceptés par la police et traduits devant la Cour du Québec, chambre criminelle et pénale, seront arrêtés et gardés sous les verrous jusqu'au moment de leur comparution devant le tribunal (66\%). C'est donc, contrairement à ce que prescrivent les différentes commissions d'étude qui se sont penchées sur la question, une majorité d'individus qui se présentent détenus devant le tribunal et ce avant même d'avoir été formellement mis en accusation.

Tableau 3

Mesure de comparution: passage de la police vers le tribunal

\begin{tabular}{lcc}
\hline & Nombre & Pourcentage \\
En liberté & 102 & $5,7 \%$ \\
Libéré par un fonctionnaire & 431 & $24,0 \%$ \\
Responsable & 76 & $4,2 \%$ \\
Mandat d'arrestation & 1186 & $66,1 \%$ \\
Mise sous garde & 1795 & $100,0 \%$ \\
Total & & \\
\hline
\end{tabular}

La cour, pour sa part, bien qu'elle choisisse de libérer 32,0 pour cent des individus prévenus au moment de leur première comparution devant le tribunal, prescrit par ailleurs l'incarcération d'un certain nombre $(3,7 \%)$ de justiciables se présentant libres à leur première comparution et impose la poursuite de la détention à 39,0 pour cent des personnes mises sous garde par les policiers. C'est donc 42,7 pour cent des personnes mises en accusation devant la Cour du Québec qui se présenteront détenues devant le juge chargé de rendre décision concernant leur affaire. Compte tenu des remarques présentées plus tôt concernant l'impact négatif de la détention sur l'issue probable des procédures et, le cas échéant, sur la sentence, et compte tenu des coûts sociaux importants non seulement pour le justiciable mais aussi pour sa famille ${ }^{39}$ et des coûts financiers tout aussi sérieux pour l'État ${ }^{40}$ qu'entraîne l'incarcération,

39. Voir à ce sujet A.P. Pires, P. Landreville et V. Blankevoort (1981), «Système pénal et trajectoire sociale», Déviance et société, vol. $5, n^{\circ} 4$, p. 319-345.

40. Voir à ce sujet le Rapport annuel de la Direction générale de la probation et des établissements de détention, Québec, Direction des communications du ministère de la Justice du Québec. 
on peut avancer que cette distribution est alarmante ou, à tout le moins, devrait l'être aux yeux des autorités concernées. D'autant plus qu'on constate que seule une minorité des personnes détenues provisoirement le sont en rapport avec des crimes contre la personne et que plus du quart des suspects écroués $(25,9 \%)$, à l'issue des procédures, seront soit libérés, soit définitivement acquittés des accusations pesant contre eux. Pour tous ceux-là, à tout le moins, on peut s'interroger sur le bien-fondé du recours à l'incarcération provisoire.

Deux situations méritent ici une attention particulière. D'abord, notons qu'en ce qui concerne les femmes appréhendées suite à la commission d'une infraction, les policiers ont plus souvent tendance que pour les hommes à ne pas les retenir en détention $(42,1 \%$ contre $28,8 \%$ sont soit laissées libres, soit remises en liberté au niveau de la police). Par contre, on constate qu'une fois mises sous garde par les policiers, ces dernières sont légèrement moins susceptibles que les hommes d'être relaxées par le juge à la comparution $(28,1 \%$ contre $32,3 \%)$. C'est donc dire que pour les femmes la mise sous garde au niveau policier risque de s'avérer particulièrement déterminante.

Une autre distribution, celle de l'usage de la détention provisoire dans les cas de violence conjugale, mérite d'être commentée. On constate, en effet, que la proportion des mises sous garde à la police, pour cette catégorie de délit, est légèrement supérieure à la proportion des mises sous garde calculée

Tableau 4

Utilisation de la détention provisoire pour la durée des procédures judiciaires

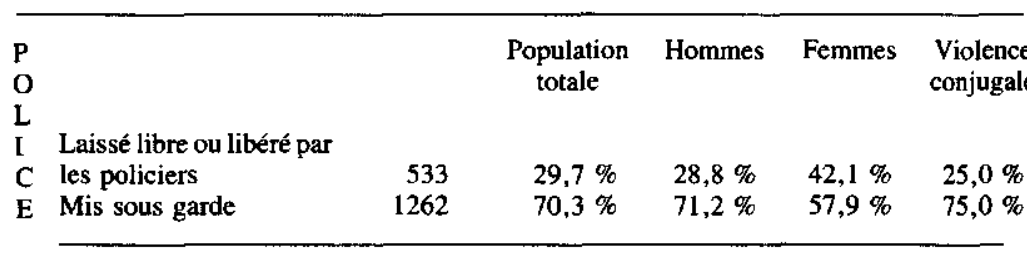

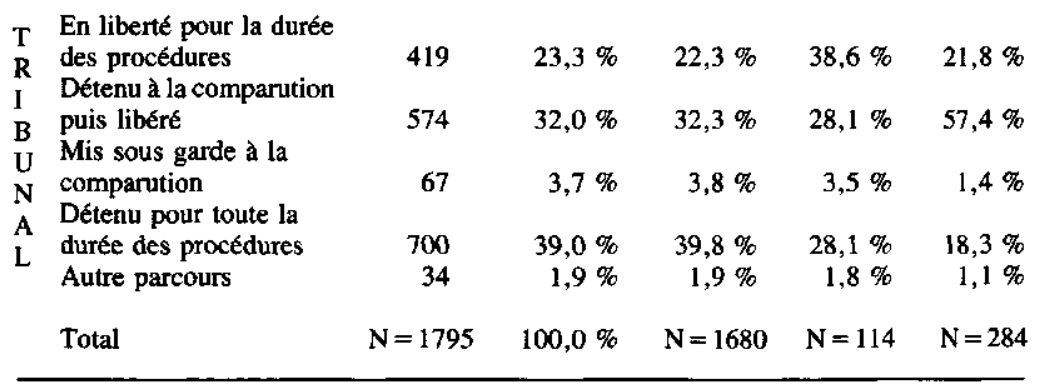


sur une base générale, sans distinction suivant la sorte d'événement (75,0\% contre $70,3 \%$ ). Toutefois, bien que les trois quarts des suspects impliqués dans des événements de cette nature se présentent détenus à la comparution, ces derniers sont, de manière marquée, plus que les autres remis en liberté par le juge à cette étape des procédures $(57,4 \%$ contre $32,0 \%)$. Plusieurs hypothèses peuvent être formulées pour expliquer cette situation. Entre autres, le profil des suspects impliqués dans ce type d'événement pourrait motiver la propension des juges à les remettre en liberté. Nos données montrent, en effet, qu'il s'agit souvent de travailleurs mariés plus âgés que la moyenne des inculpés et qui ne possèdent pas d'antécédents judiciaires. En outre, il s'agit d'un type de cause particulièrement susceptible d'être affectée par la volonté de la victime de poursuivre ou non les procédures, celle-ci étant souvent, dans ce cas, le seul témoin à charge, le seul susceptible de fournir la preuve nécessaire à l'établissement de la culpabilité de l'accusé. Il s'agit très certainement là d'une problématique qui mérite d'être fouillée plus à fond.

\section{UNE NOUVELLE FAÇON DE CONCEVOIR L'ACTIVITÉ DU TRIBUNAL}

Brodeur ${ }^{41}$ (1980, p. 15), constatait: «Le système de la justice pénale paraît trouver sa pierre d'assise dans les décisions que rendent les tribunaux au cours d'exercices ritualisés qui ont pour nom "procès" et où s'incarne de façon privilégiée la pratique du droit criminel, c'est-à-dire l'obligation qui est faite aux instances de la poursuite de défaire la présomption d'innocence dont jouit légalement le prévenu.» Or, notait encore l'auteur: «les procès, si l'on compare le nombre de leurs occurrences à la masse des affaires traitées par la justice pénale, sont relativement rares» (p. 16). Aux États-Unis, le même constat avait fait dire à Feeley ${ }^{42}(1979$, p. 186): «In fact, trials are so rare that some prosecutors and judges regard them as a welcome change of pace». À l'instar de ces auteurs, nous constatons qu'encore aujourd'hui, à la Cour du Québec, la tenue de procès en bonne et due forme constitue beaucoup plus l'exception que la règle. Ainsi, à l'étude de notre échantillon, on constate que les juges ont été appelés à rendre verdict concernant la culpabilité d'un inculpé dans seulement 13,4 pour cent des cas portés à leur attention. Dans une majorité de situations $(65,4 \%)$, les inculpés ont reconnu d'euxmêmes leur culpabilité: ils ont plaidé coupables (schéma 1).

41. J.-P. Brodeur (1980), «Le trafic des plaidoyers dans la justice pénale: le verbe "dealer" de l'anglais "to deal"," Les Cahiers du socialisme, $\mathrm{n}^{\circ} 5$.

42. M.M. Feeley (1979), The Process is the Punishment, New York, Russell Sage Foundation. 


\section{Schêma 1}

Décisions rendues par le tribunal

Cour du Québec, chambre criminelle et pénale district judiciaire de Montréal, 1989

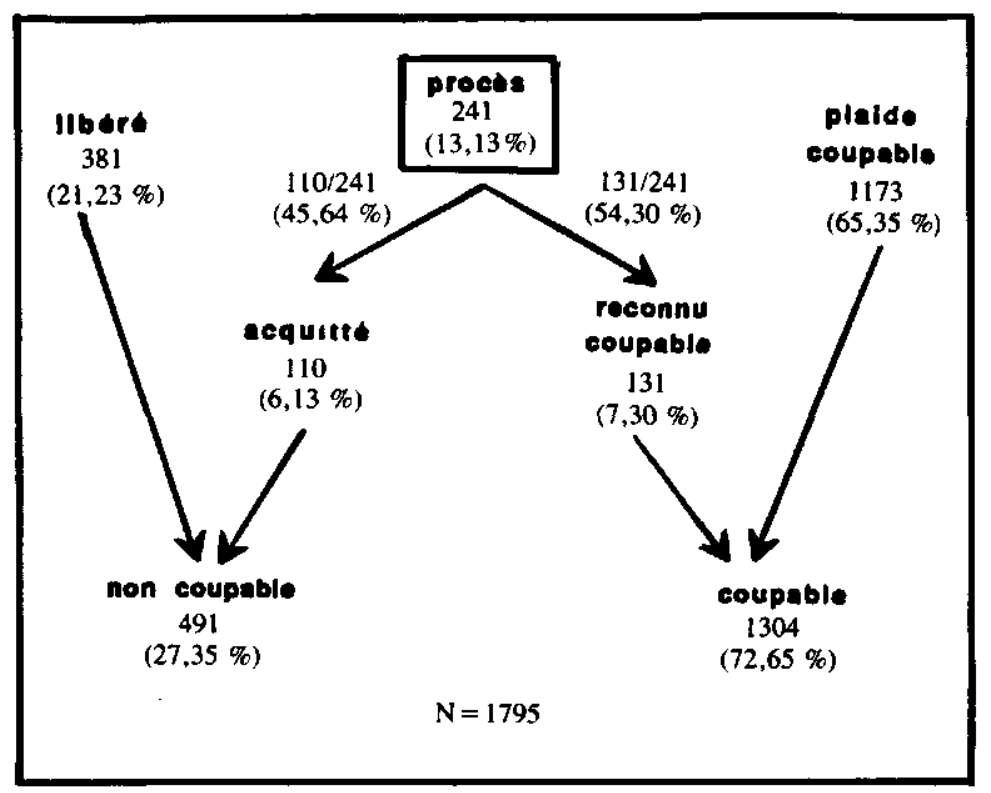

Cette image nous oblige à repenser l'idée que l'on se fait de l'activité du tribunal. Les procès à grand déploiement, que s'évertuent à nous présenter les médias, sont beaucoup plus rares qu'on veut bien le laisser entendre. Par contre, il serait erroné de prétendre que l'activité verdictive des juges se restreint aux 13,4 pour cent de dossiers qui donnent lieu à un véritable procès. Il est une catégorie de décisions qui revient directement au juge qu'on a trop souvent tendance à oublier. Il s'agit des dossiers où, appelé à statuer sur la culpabilité factuelle et légale de l'inculpé, celui-ci se voit contraint de le libérer $(21,2 \%$ des cas) car il estime que la preuve qui lui est présentée ne lui permet pas de conclure définitivement à la responsabilité du suspect ou, à l'inverse, à son innocence. Cet acte, au même titre que le fait de statuer sur la culpabilité du contrevenant, constitue également, de notre point de vue, un verdict. Cette décision n'est toutefois pas définitive. L'accusé n'est pas irrévocablement «absous de ses fautes»; il pourra encore, advenant de nouveaux développements dans le dossier, être rappelé devant le tribunal. La porte demeure ouverte.

Reste qu'une grande partie du travail des juges est essentiellement de nature sentencielle. En effet, puisque 65,4 pour cent des inculpés reconnaissent 
leur culpabilité à un certain nombre ou à la totalité des accusations formulées à leur encontre et que 7,3 pour cent des suspects sont reconnus coupables, il s'ensuit que les juges doivent rendre sentence dans 72,7 pour cent des affaires sur lesquelles ils sont appelés à statuer.

\section{PLAIDER COUPABLE OU «RISQUER» LE PROCÈS}

Au moment de dresser la liste des incitatifs susceptibles de pousser un inculpé à enregistrer un plaidoyer de culpabilité, on oppose la certitude concernant l'issue de la cause, découlant d'une entente intervenue entre le procureur de la poursuite et l'avocat de la défense, au risque du procès: «un tiens vaut mieux que deux tu l'auras ${ }^{43}$ » (Gravel et Cousineau, 1989); «vaut mieux le pire arrangement que le meilleur procès ${ }^{44}$ " (Brodeur, 1980) constitueraient des leitmotivs, partagés par les suspects traduits devant les tribunaux ${ }^{45}$. Qu'en est-il véritablement des risques du procès? Parmi ceux qui ont choisi de soumettre leur cause à l'arbitrage, une proportion légèrement supérieure $(54,4 \%)$ ont été reconnus coupables, les autres $(45,6 \%)$ se voyant définitivement acquittés des charges retenues contre eux.

Un autre incitatif à plaider coupable résiderait dans le fait que l'enregistrement d'un plaidoyer de culpabilité raccourcit de manière significative la longueur des procédures: «La longueur de la procédure et conséquemment les coûts élevés des avocats minent la résistance des justiciables ${ }^{46}$ », concluent Pires et al. (1981, p. 337).

Bon nombre de préjugés circulent concernant la longueur des procédures judiciaires. Les uns estiment que la justice est expédiée, qu'il suffit souvent de quelques minutes pour envoyer quelqu'un «au trou» pour une éternité. Les autres tablent au contraire sur la tenue générale de procès interminables, étirés à l'excès. Dans une certaine mesure, les uns comme les autres ont tantôt raison, tantôt tort. Il est des procédures qui se bouclent en une journée (lorsque le prévenu plaide coupable dès le moment de sa première comparution, ce qui arrive dans 3,5 pour cent des cas de notre échantillon), et il est aussi des affaires qui s'éternisent (les procès devant jury, entre autres). La durée

43. Gravel et Cousineau (1989), La Pratique de la négociation de plaidoyer au palais de justice de Montréal, Montréal, Centre international de criminologie de Montréal.

44. Loc. cit., note 39.

45. Cette assurance est néanmoins toute relative. La négociation de plaidoyer consistant en une pratique informelle, nullement encadrée, il s'ensuit que l'inculpé qui accepte de négocier sa cause ne possède aucune garantie que l'entente intervenue entre la défense et la poursuite sera respectée. En outre, le juge reste maître de la décision finale concernant la sentence et, à ce titre, ne se trouve nullement lié par les suggestions des avocats.

46. Loc. cit., note 37 . 
moyenne des procédures s'inscrit toutefois quelque part entre ces deux extrêmes: selon nos données, elle se situerait autour de 3 mois et demi avec un écart type de 1'ordre de 90 jours et une médiane située à 80 jours. Soixantequinze pour cent des cas que nous étudions se trouvent réglés en moins de 5 mois, alors que seule une petite minorité $(1,2 \%)$ s'étirent au-delà d'une année ${ }^{47}$...

Le plaidoyer de culpabilité de même que la libération de l'accusé s'avèrent sans contredit les causes les plus rapidement réglées (moyenne : 95 jours dans chacun des cas; écart type de 89 jours dans le cas du plaidoyer de culpabilité, 74 jours en ce qui a trait à la décision de libérer l'accusé). La tenue d'un procès augmente substantiellement la durée des procédures (durée moyenne dans le cas des procès à l'issue desquels l'accusé est acquitté de 154 jours avec un écart type de 97 jours et de 160 jours avec un écart type de 85 jours dans le cas où l'accusé est formellement reconnu coupable). La durée des procédures est donc sans aucun doute liée au cheminement qu'emprunte la plainte.

\section{CONCLUSION}

De l'activité des tribunaux criminels, et plus largement des tribunaux pénaux, il y a beaucoup à dire. Si l'on élargit le champ de nos connaissances de manière à y introduire la dimension relations inter-agences, il y a encore plus à dire. Les quelques idées exposées dans le cadre de cet article sont loin d'épuiser le sujet. Elles ne constituent même pas un prolégomène suffisant.

En effet, l'ensemble des étapes à travers lesquelles doit passer le justiciable accusé d'une infraction peut être conçu comme une course à obstacles. Les agents pénaux impliqués (policiers, procureurs de la Couronne, juges...) prennent à tour de rôle des décisions concernant chaque affaire soumise à leur attention (jugement sur le fondement de la plainte, décision de poursuivre l'enquête, de porter la plainte devant les tribunaux, de mettre sous garde l'ac-

47. Le fait que nos données ne concernent que les dossiers clos au moment de l'étude peut laisser supposer que cette moyenne serait plus ou moins sous-évaluée. Une étude préalable concernant les dossiers traités entre 1982 et 1986 à la Cour du Québec (à l'époque Tribunal des sessions de la paix) đu district judiciaire de Montréal montre qu'invariablement moins de 1 pour cent des causes entendues par ce tribunal prennent plus d'une année à se régler (M.-M. Cousineau, 1990, De la théorie à la pratique: l'examen statistique des pratiques de la Cour des sessions de la paix du district judiciaire de Montréal, 1982-1986, rapport de recherche inédit). On peut donc prétendre que la moyenne calculée ici, bien qu'effectivement sous-évaluée, reste malgré tout très près de la réalité. Il n'est par ailleurs pas exclu que ce 1 pour cent des causes se particularise franchement. Nous ne sommes pas à même de conclure. Toutefois, sur le 99 pour cent des causes restantes, nous pouvons nous prononcer de manière certaine. 
cusé, au niveau de la police; décision de garder en détention préventive ou de relâcher le suspect à différentes étapes de la procédure, citation à procès, décision concernant l'issue de la cause, imposition d'une sentence le cas échéant, au niveau du tribunal...). À tour de rôle et combinées, on peut supposer que l'agencement de ces décisions contribue à définir des trajectoires particulières pour les contrevenants mis en accusation.

On conçoit, ainsi que le remarquent très pertinemment Duchastel et Laberge $^{48}$ (1989), qu'il existe un niveau minimum de cohérence entre les agences impliquées, cohérence minimale qui permet de penser la chose pénale comme système: «[...] chacune des agences est fondée directement par et dans le droit, produit de la nécessité pour l'État de perpétuer l'ordre et de réprimer le crime. À ce titre, les agences peuvent être pensées comme parties essentielles d'un tout qui les constitue de façon cohérente» (p. 6). En outre, poursuivent Duchastel et Laberge: «les modalités de circulation à travers les agences ne sont pas aléatoires, elles se produisent selon un certain ordre. Ces modalités processuelles sont régies par des règles procédurales qui en déterminent la mécanique générale [...] [mais] n'impliquent nullement que tous les individus soient traités de la même façon à l'intérieur de ce processus partiellement réglé» (p. 8). C'est toute une sociologie du processus pénal, alimentée par son fonctionnement empirique, qui se profile derrière ces constats. Celle-ci mérite certainement d'être développée plus avant.

48. J. Duchastel et D. Laberge (1989), «Bénévolence et délation: la transformation des modes étatiques de contrôle social», Colloque sur État et société civile, Ottawa. 